\title{
Effect of Axial Porosities on Flexomagnetic Response of In-Plane Compressed Piezomagnetic Nanobeams
}

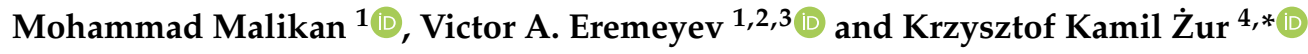 \\ 1 Department of Mechanics of Materials and Structures, Faculty of Civil and Environmental Engineering, \\ Gdansk University of Technology, 80-233 Gdansk, Poland; mohammad.malikan@pg.edu.pl (M.M.); \\ victor.eremeev@pg.edu.pl (V.A.E.) \\ 2 Laboratory of Mechanics of Biomaterials, Research and Education Center "Materials", Don State Technical \\ University, Gagarina sq., 1, 344000 Rostov on Don, Russia \\ 3 Department of Civil and Environmental Engineering and Architecture (DICAAR), University of Cagliari, \\ Via Marengo, 2, 09123 Cagliari, Italy \\ 4 Faculty of Mechanical Engineering, Bialystok University of Technology, 15-351 Bialystok, Poland \\ * Correspondence: k.zur@pb.edu.pl
}

Received: 29 October 2020; Accepted: 23 November 2020; Published: 24 November 2020

\begin{abstract}
We investigated the stability of an axially loaded Euler-Bernoulli porous nanobeam considering the flexomagnetic material properties. The flexomagneticity relates to the magnetization with strain gradients. Here we assume both piezomagnetic and flexomagnetic phenomena are coupled simultaneously with elastic relations in an inverse magnetization. Similar to flexoelectricity, the flexomagneticity is a size-dependent property. Therefore, its effect is more pronounced at small scales. We merge the stability equation with a nonlocal model of the strain gradient elasticity. The Navier sinusoidal transverse deflection is employed to attain the critical buckling load. Furthermore, different types of axial symmetric and asymmetric porosity distributions are studied. It was revealed that regardless of the high magnetic field, one can realize the flexomagnetic effect at a small scale. We demonstrate as well that for the larger thicknesses a difference between responses of piezomagnetic and piezo-flexomagnetic nanobeams would not be significant.
\end{abstract}

Keywords: flexomagneticity; stability analysis; Euler-Bernoulli beam; porous nanobeam

\section{Introduction}

Flexomagneticity arises through elastic strain gradient or magnetic field gradient during electric magnetization in the magneto-elastic coupling in smart structures and actuators [1-3]. Such an effect should be significant in nano electro-mechanical systems (NEMS) and other smart sensors and actuators. Similar to this influence, viz. flexoelectricity for centrosymmetric and non-centrosymmetric structures has been estimated widely [4-19]. However, the flexomagneticity effect has been less known. Although the structure and physics of a flexomagneticity phenomenon are very complicated, it has economic implications. Physically, it may be difficult to interpret, but the basic idea satisfactorily shows the importance of flexomagneticity.

In discussing a mechanical response of nanomaterials with magneto-mechanical coupling, the importance of piezomagneticity has been profoundly evaluated by scholars in the contemporary decade [20-39]. However, fewer studies are available regarding flexomagneticity [40-45]. In the available literature, Sidhardh and Ray [40] worked on the bending response of a thin cantilever nanobeam with flexomagnetic property. They discussed both direct and reverse impacts of magneto-elastic coupling with the presence of the surface elasticity. Zhang et al. [41] conducted the importance of flexomagneticity for a nanoscale size-dependent Euler-Bernoulli beam exposed to 
transverse static loading. To present the size-dependent mechanical behavior of the structure, they utilized the surface elasticity. Both direct and converse flexomagnetic influences were investigated when the nano-sized beam was kept in ends with fixed, pivot, and free edge conditions. One of their momentous achievements was the conclusion that the flexomagneticity is a scale-dependent property of materials. Recently, Malikan and Eremeyev [42] investigated vibrating nanobeams by taking into account the piezomagnetic, particularly flexomagnetic, properties. The free vibration frequencies were evaluated by linear assumptions of strain and the impact of size-dependency was inspected on the basis of a new nonlocal elasticity theory. The size-dependent behavior of the flexomagnetic effect was affirmed by their results. In another effort, Malikan and Eremeyev [43] explored the flexomagnetic response of a smart nanobeam in a vibrational condition based on large frequency modes. The small scale response of the nanoscale beam was searched by imposing a nonlocal strain gradient elasticity approach into the constitutive equations. Besides these, Malikan and Eremeyev [44] performed research on non-linear static bending of smart nanoscale beams while the material included a remarkable flexomagnetic response. The computational model was solved by coupling between an analytical and numerical solution method. The new finding demonstrated that the presence of the flexomagnetic feature leads to diminishing the deflections. More recently, Malikan et al. [45] examined post-buckling stability of a nanoparticle in which both flexomagnetic and piezomagnetic properties were included. The calculations extracted new findings that are helpful for magnetic nanosensors applications.

To the best of our knowledge, the flexomagnetic studies on the mechanical response of nanostructures referred to above represent the majority of cases in the literature, unless otherwise stated. In these references, the models are restricted to piezomagnetic nanobeams under some mechanical analyses. The present research attempts to demonstrate the flexomagnetic property for the stability problem of a nano-sized beam, while it includes a material imperfection with intentional nonlocality and size-dependent characteristics according to the nonlocal strain gradient constitutive equation. The structural and material imperfection is estimated in the framework of different types of porosities. The nonlocal influences that can affect the flexomagnetic response of the Euler-Bernoulli nanoscale beam are addressed in this paper. A sinusoidal transverse deflection is assumed when applying the Navier approach to beam flexure. Terminally, the nanobeam is considered for variations of key parameters based on the three cases, i.e., a simple nanobeam, piezomagnetic nanobeam, and piezo-flexomagnetic nanobeam.

\section{Formulation of the Problem}

\subsection{Constitutive Relations for Piezo-Flexomagnetic Solids}

Pursuing [1-3], we briefly introduce constitutive relations for flexomagnetic material. In the following, we assume infinitesimal deformations under isothermal conditions. Therefore, the variables are the displacements $u$ and the magnetic field is $H$ as below

$$
u=u(x), H=H(x) .
$$

where $x$ is a position vector, and $H$ is a tensor of first-order. Within the flexomagneticity, the free energy density function $U$ has the form

$$
\begin{aligned}
& U=U(\varepsilon, \eta, H)=-\frac{1}{2} H \cdot a \cdot H+\frac{1}{2} \varepsilon: C: \varepsilon+\frac{1}{2} \eta: g: \eta+\varepsilon: r: \eta \\
& -H \cdot q: \varepsilon-H \cdot f: \eta
\end{aligned}
$$

where $\varepsilon$ is the strain tensor and its gradient is

$$
\varepsilon=\frac{1}{2}\left(\nabla u+\nabla u^{T}\right), \eta=\nabla \varepsilon
$$


where $\nabla$ is the 3D nabla-operator in a general case. We introduced to Equation (2) several tensors of material parameters. $q$ is the third-order piezomagnetic tensor, $a$ is the second-order magnetic permeability tensor, $g$ is the sixth-order gradient elasticity tensor, $C$ is the fourth-order elasticity coefficient tensor, $f$ is the fourth-order flexomagnetic tensor, and the strain and strain-gradient tensors are coupled by $r$, which itself is a fifth-order tensor. Moreover, ".", “:”, and “:” stand for scalar (inner) products in spaces of vectors, second-order and third-order tensors, respectively.

In the following, we express $H$ through the magnetic potential $\psi[40,41]$,

$$
H=-\nabla \psi
$$

The governing equations of the static flexomagneticity can be derived using the virtual work principle as

$$
\delta \Pi=\delta A .
$$

in which $\Pi=\int_{V} U d V$, where $V$ is the volume of domain that occupies the flexomagnetic solid and $\delta A$ is the work of external loads.

For simplicity, we assume the simple form of

$$
\delta A=\int_{V} F \cdot \delta u+\int_{\partial V} t \cdot \delta u d s .
$$

where $F$ and $t$ are external mass forces and surface traction.

Using the standard calculus of variations from Equation (5) we get

$$
\begin{gathered}
\nabla \cdot(\sigma-\nabla \cdot \xi)+F=0, \\
\nabla \cdot B=0 .
\end{gathered}
$$

in which $B$ is the magnetic induction vector. The following constitutive equations are introduced

$$
\begin{gathered}
\sigma=\frac{\partial U}{\partial \varepsilon} \equiv C: \varepsilon+r: \eta-H \cdot q, \\
\xi=\frac{\partial U}{\partial \eta} \equiv g: \eta+\varepsilon: r-H \cdot f, \\
B=-\frac{\partial U}{\partial H}=a \cdot H+q: \varepsilon+f: \eta .
\end{gathered}
$$

\subsection{The Piezo-Flexomagnetic Beam Model}

The piezo-flexomagnetic nanobeam studied in this research is exhibited in Figure 1. In the figure, length and height of the beam are denoted by $L$ and $h$, respectively. 


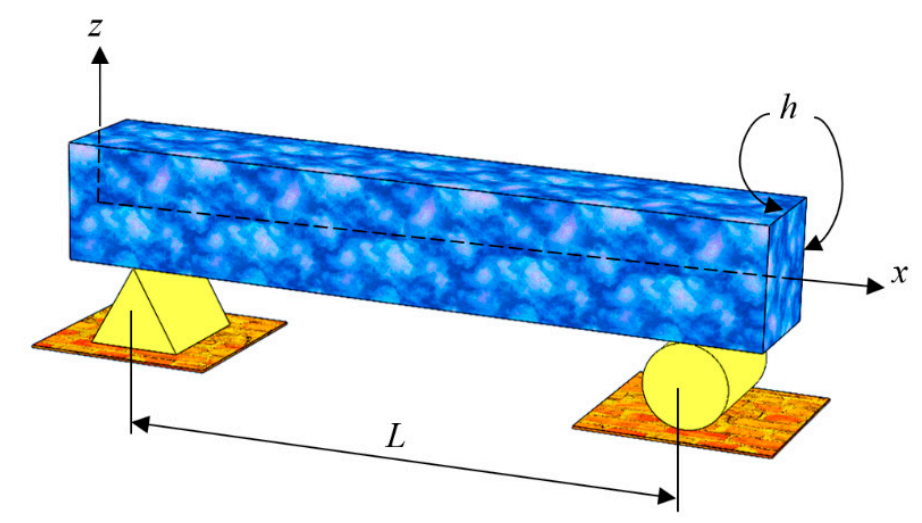

Figure 1. Geometry and description of a continuum nanobeam as a square actuator installed on simple end conditions.

The constitutive equations (Equation (8)) can be re-written for a beam incorporating the flexomagnetic property as $[40,41]$

$$
\begin{gathered}
\sigma_{x x}=C_{11}^{p} \varepsilon_{x x}-q_{31} H_{z}, \\
\xi_{x x z}=g_{31} \eta_{x x z}-f_{31} H_{z}, \\
B_{z}=a_{33} H_{z}+q_{31} \varepsilon_{x x}+f_{31} \eta_{x x z} .
\end{gathered}
$$

in which $\eta_{x x z}$ and $\varepsilon_{x x}$ are the gradient of the axial elastic strain and the strain itself, $C_{11}=C_{1111}$ is the elastic modulus, $\sigma_{x x}$ is the axial stress, $f_{31}=f_{3311}$ denotes the component of the fourth-order flexomagnetic coefficients tensor, $a_{33}$ represents the component of the second-order magnetic permeability tensor, $q_{31}=q_{311}$ depicts the component of the third-order piezomagnetic tensor, $\xi_{x x z}$ is the component of the higher-order hyper stress tensor and is an induction of coverse flexomagnetic effect, $B_{z}$ and $H_{z}$ exhibit the magnetic flux and the component of magnetic field, respectively, and $g_{31}=g_{311311}$ illustrates the influence of the sixth-order gradient elasticity tensor. It is worth mentioning that the piezomagnetic tensor would be non-zero for non-centrosymmetric ferroics only, but the flexomagnetic tensor would be non-zero for both centrosymmetric and non-centrosymmetric materials.

The displacement field with respect to the Euler-Bernoulli beam is available as [46-48]

$$
\begin{gathered}
u_{1}(x, z)=u(x)-z \frac{d w(x)}{d x} \\
u_{3}(x, z)=w(x) .
\end{gathered}
$$

where $u_{i}(i=1,3)$ represent the points' displacements in direction of $x$ and $z, u$ and $w$ are the axial and transverse displacements of the mid-plan, respectively, see Figure 1. To show the thickness coordinate, the $z$ parameter is used.

Due to the linear problem studied in this research, the linear Lagrangian strain can be employed as

$$
\varepsilon_{i j}=\frac{1}{2}\left(\frac{\partial u_{i}}{\partial x_{j}}+\frac{\partial u_{j}}{\partial x_{i}}\right)
$$


The components of the transverse strain and the strain gradient can be presented on the basis of substituting Equation (12) into Equation (13) as below

$$
\begin{gathered}
\varepsilon_{x x}=\frac{d u}{d x}-z \frac{d^{2} w}{d x^{2}}, \\
\eta_{x x z}=\frac{d \varepsilon_{x x}}{d z}=-\frac{d^{2} w}{d x^{2}}, \\
\eta_{x x x}=\frac{d \varepsilon_{x x}}{d x}=-z \frac{d^{3} w}{d x^{3}} .
\end{gathered}
$$

As the $\eta_{x x x}$ is small compared to the $\eta_{x x z}$, it can be ignored. With respect to the Lagrange's principle, we have

$$
\delta \int\left(\Pi_{W}+\Pi_{U}\right)=0 .
$$

in which $\Pi_{W}$ and $\Pi_{U}$ depict the performed work by outer loads, and the total internal strain energy (magnetic potential energy and mechanical strain energy). The total strain energy by means of Equation (14) can be demonstrated as

$$
\delta \Pi_{U}=\int_{V}\left(\sigma_{x x} \delta \varepsilon_{x x}+\xi_{x x z} \delta \eta_{x x z}-B_{z} \delta H_{z}\right) d V .
$$

One can obtain the governing equation and non-classical boundary conditions as below

$$
\begin{gathered}
\delta \Pi_{U_{1}}^{M e c h}=-\int_{0}^{L}\left(\frac{d N_{x}}{d x} \delta u+\frac{d^{2} M_{x}}{d x^{2}} \delta w+\frac{d^{2} T_{x x z}}{d x^{2}} \delta w\right) d x, \\
\delta \Pi_{U_{2}}^{M a g}=-\int_{0}^{L} \int_{-h / 2}^{h / 2} \frac{d B_{z}}{d z} \delta \Psi d z d x, \\
\delta \Pi_{U_{1}}^{M e c h}=\left.\left(N_{x} \delta u-M_{x} \frac{d \delta w}{d x}-T_{x x z} \frac{d \delta w}{d x}+\frac{d M_{x}}{d x} \delta w+\frac{d T_{x x z}}{d x} \delta w\right)\right|_{0} ^{L}, \\
\delta \Pi_{U_{2}}^{M a g}=\left.\int_{0}^{L}\left(B_{z} \delta \Psi\right)\right|_{-h / 2} ^{h / 2} d x .
\end{gathered}
$$

in which

$$
\begin{gathered}
N_{x}=\int_{-h / 2}^{h / 2} \sigma_{x x} d z, \\
M_{x}=\int_{-h / 2}^{h / 2} \sigma_{x x} z d z, \\
T_{x x z}=\int_{-h / 2}^{h / 2} \xi_{x x z} d z .
\end{gathered}
$$


The performed work by external factors can be expressed as below $[49,50]$

$$
\Pi_{W}=\frac{1}{2} \int_{0}^{L} N_{x}^{0}\left(\frac{d w}{d x}\right)^{2} d x .
$$

Its first variational form is

$$
\delta \Pi_{W}=\int_{0}^{L} N_{x}^{0}\left(\frac{d \delta w}{d x} \frac{d w}{d x}\right) d x
$$

in which $N_{x}^{0}$ shows the axial membrane load.

Hereafter, the magnetic field's transverse component can be expressed as

$$
H_{z}+\frac{d \Psi}{d z}=0
$$

Assuming the condition of a closed circuit as well as the inverse piezomagnetic effect, one gives the magnetic boundary conditions as

$$
\begin{aligned}
& \Psi\left(+\frac{h}{2}\right)=\psi, \\
& \Psi\left(-\frac{h}{2}\right)=0 .
\end{aligned}
$$

where $\psi$ determines the external magnetic potential applied to the upper surface of the beam. With mixing Equations (11), (17b), (18b), (24) and (25), one can derive the magnetic polarization and magnetic field as $[40,41]$

$$
\begin{gathered}
\Psi=-\frac{q_{31}}{2 a_{33}}\left(z^{2}-\frac{h^{2}}{4}\right) \frac{d^{2} w}{d x^{2}}+\frac{\psi}{h}\left(z+\frac{h}{2}\right), \\
H_{z}=z \frac{q_{31}}{a_{33}} \frac{d^{2} w}{d x^{2}}-\frac{\psi}{h} .
\end{gathered}
$$

The nanoscale atomic interactions can be projected in a continuum space by means of the nonlocal strain gradient elasticity theory (NSGT) given as [51]

$$
\left(1-\mu \frac{d^{2}}{d x^{2}}\right) \sigma_{x x}^{N L}=\left(1-l^{2} \frac{d^{2}}{d x^{2}}\right) \sigma_{x x}^{L} .
$$

In order to allocate the influence of nonlocality, namely stiffness-softening, the nonlocal parameter $\mu$ (unit: square nanometers $\left.=(n m)^{2}\right)$ is employed. Note that $\mu(n m)^{2}=\left(e_{0} a\right)^{2}$, in which $e_{0}$ and $a$ are two small scale factors that determine the nonlocal parameter. Furthermore, in order to set the effect of the size deduction, namely stiffness-hardening, the $l(n m)$ parameter is used, which is named as the strain gradient length scale parameter. It is to be noted that $[52,53]$ confirmed the fact that the aforesaid small scale factors are not material constants and can be varied by respecting a variety of conditions. Additionally, the $N L$ and $L$ indexes respectively express the nonlocal and the local components of stress.

Thus, inserting Equations (26)-(28) into Equations (9)-(11), it is possible to present the stress field component, the higher-order moment stress tensor component, and magnetic induction component in the NSGT form as

$$
\begin{gathered}
\left(1-\mu \frac{d^{2}}{d x^{2}}\right) \sigma_{x x}=\left(1-l^{2} \frac{d^{2}}{d x^{2}}\right)\left[C_{11}^{p} \frac{d u}{d x}-z\left(C_{11}^{p}+\frac{q_{31}^{2}}{a_{33}}\right) \frac{d^{2} w}{d x^{2}}+\frac{q_{31} \psi}{h}\right] \\
\left(1-\mu \frac{d^{2}}{d x^{2}}\right) \xi_{x x z}=\left(1-l^{2} \frac{d^{2}}{d x^{2}}\right)\left[-\left(g_{31}+\frac{q_{31} f_{31} z}{a_{33}}\right) \frac{d^{2} w}{d x^{2}}+\frac{f_{31} \psi}{h}\right]
\end{gathered}
$$




$$
\left(1-\mu \frac{d^{2}}{d x^{2}}\right) B_{z}=\left(1-l^{2} \frac{d^{2}}{d x^{2}}\right)\left(-f_{31} \frac{d^{2} w}{d x^{2}}-\frac{a_{33} \psi}{h}\right)
$$

Hence, on the basis of Equations (29)-(31), Equations (19)-(21) can be expanded as [54-61]

$$
\begin{gathered}
\left(1-\mu \frac{d^{2}}{d x^{2}}\right) N_{x}=\left(1-l^{2} \frac{d^{2}}{d x^{2}}\right)\left(C_{11}^{p} h \frac{d u}{d x}+q_{31} \psi\right) \\
\left(1-\mu \frac{d^{2}}{d x^{2}}\right) M_{x}=\left(1-l^{2} \frac{d^{2}}{d x^{2}}\right)\left(-I_{z}\left(C_{11}^{p}+\frac{q_{31}^{2}}{a_{33}}\right) \frac{d^{2} w}{d x^{2}}\right), \\
\left(1-\mu \frac{d^{2}}{d x^{2}}\right) T_{x x z}=\left(1-l^{2} \frac{d^{2}}{d x^{2}}\right)\left(-g_{31} h \frac{d^{2} w}{d x^{2}}+f_{31} \psi\right) .
\end{gathered}
$$

in which $I_{z}=\int_{A} z^{2} d A$ is the moment of inertia.

Due to inevitable variations in the manufacturing processes, the presence of some porosity in nanobeams is unavoidable. Inclusion of this imperfection into mechanical analysis of the piezomagnetic-flexomagnetic nano-sized beam is performed as [62]

$$
C_{11}^{p}=C_{11}\left(\frac{1.21-\alpha \lambda(x)}{1.21}\right)^{2.3}
$$

where $\alpha$ denotes the porosity coefficient. Axial porosities are defined mathematically and analytically in Table 1 [62].

Table 1. Axial porosity distribution patterns.

\begin{tabular}{ccc}
\hline Porosity Type & $\lambda(x)$ & Ranges of $\alpha$ \\
\hline “O" type distribution & $\eta_{1}^{2} \sin \left(\frac{\pi}{L} x\right)$ & $0 \leq \alpha<0.344$ \\
" $\overline{\mathrm{O}}$ " type distribution & $\eta_{2}^{2}\left[1-\sin \left(\frac{\pi}{L} x\right)\right]$ & $0 \leq \alpha<0.112$ \\
" $\mathrm{X}$ " type distribution & $\eta_{1} \eta_{2} \sin \left(\frac{\pi}{L} x\right)$ & $0 \leq \alpha<0.197$ \\
" $\overline{\mathrm{X}}$ " type distribution & $\eta_{1} \eta_{2}\left[1-\sin \left(\frac{\pi}{L} x\right)\right]$ & $0 \leq \alpha<0.197$ \\
Uniform type distribution & 1 & $0 \leq \alpha<0.85$ \\
\hline \multicolumn{2}{c}{$\eta_{1}=\frac{\pi}{2}, \eta_{2}=\frac{\pi}{\pi-2}$} \\
\hline
\end{tabular}

On the basis of Equations (17a) and (23), and replacing into Equation (15), one can derive the local governing relations as below

$$
\begin{gathered}
\frac{d N_{x}}{d x}=0, \\
\frac{d^{2} M_{x}}{d x^{2}}+\frac{d^{2} T_{x x z}}{d x^{2}}+N_{x}^{0} \frac{d^{2} w}{d x^{2}}=0 .
\end{gathered}
$$

Equations (36) and (37) are decoupled and thus, Equation (37) gives the stability equation in order to have values of critical buckling loads.

Thus, we should transfer the local stability equation (Equation (37)) to a size-dependent relation. In so doing, inserting Equation (37) into Equation (33), one gets

$$
M_{x}=-\mu\left(\frac{d^{2} T_{x x z}}{d x^{2}}+N_{x}^{0} \frac{d^{2} w}{d x^{2}}\right)-I_{z}\left(C_{11}^{p}+\frac{q_{31}^{2}}{a_{33}}\right)\left(1-l^{2} \frac{d^{2}}{d x^{2}}\right) \frac{d^{2} w}{d x^{2}} .
$$

Then, Equation (37) can be re-derived by mixing Equation (38) and Equation (34) as follows

$$
\left(1-\mu \frac{d^{2}}{d x^{2}}\right)\left(B \frac{d^{4} w}{d x^{4}}+N_{x}^{0} \frac{d^{2} w}{d x^{2}}\right)+D\left(1-l^{2} \frac{d^{2}}{d x^{2}}\right) \frac{d^{4} w}{d x^{4}}=0 .
$$


in which $B=-g_{31} h$, and $D=-I_{z}\left(C_{11}^{p}+\frac{q_{31}^{2}}{a_{33}}\right)$.

Here, it is required to define the pre-buckling compression as membrane loads as

$$
N_{x}^{0}=N^{M e c h}+N^{M a g} .
$$

where $N^{\text {Mech }}$ and $N^{M a g}$ are the axial membrane magnetic and mechanical loads assumed as follows:

$$
\begin{gathered}
N^{M e c h}=-P_{c r}, \\
N^{M a g}=-q_{31} \psi .
\end{gathered}
$$

\section{Solution of the Problem}

Regarding the analytical closed-form solution, we apply the following transverse deflection equation

$$
w(x)=\sum_{m=1}^{\infty} X_{m}(x)
$$

in which $X_{m}$ is an allowable function satisfying the boundary conditions of simply-simply supported (S-S) for two ends.

The dedicated kinematic and nonlocal strain gradient constitutive boundary conditions for the S-S nanobeam are expressed by Table 2 [63-66]

Table 2. Constitutive boundary conditions for S-S.

\begin{tabular}{cc}
\hline $\begin{array}{c}\text { Nonlocal Strain Gradient Conditions at } \\
(\mathbf{0}, \mathbf{L})\end{array}$ & $\begin{array}{c}\text { Local Conditions } \\
(l=\mu=\mathbf{0}) \text { at }(\mathbf{0}, \mathbf{L})\end{array}$ \\
\hline$w=0$ & $w=0$ \\
$M_{n l}=\left(1+l^{2} \frac{d^{2}}{d x^{2}}\right) M_{l}+\mu \frac{d}{d x}\left(\frac{d^{2} T_{x x z}}{d x^{2}}+N_{x}^{0} \frac{d^{2} w}{d x^{2}}\right)=0^{*}$ & $M_{l}=-D \frac{d^{2} w}{d x^{2}}=0 *$ \\
$T_{x x z}=B \frac{d^{2} w}{d x^{2}}+f_{31} \psi=0$ & $T_{x x z}=B \frac{d^{2} w}{d x^{2}}+f_{31} \psi=0$ \\
\hline${ }^{*}$ Sub-indexes $(n l$ and $l)$ are nonlocal and local phases, respectively.
\end{tabular}

The pointed conditions in Table 2 may be satisfied by the admissible function given below [57]

$$
X_{m}(x)=\sin \left(\alpha_{m} x\right)
$$

in which $\alpha_{m}=\frac{m \pi}{L}$.

Substituting Equation (42) into Equation (39), the closed-form size-dependent buckling relation for the piezo-flexomagnetic nanobeam becomes

$$
P_{c r}=\int_{0}^{L} \frac{-\left(B \mu+l^{2} D\right) \alpha_{m}^{6}+\left(B-q_{31} \psi \mu+D\right) \alpha_{m}^{4}+q_{31} \psi \alpha_{m}^{2}}{\mu \alpha_{m}^{4}-\alpha_{m}^{2}} \Upsilon_{m} d x .
$$

in which $Y_{m}$ is a residue. It is important to bear in mind that all of the results of the present work are given for $m=1$.

\section{Numerical Results}

\subsection{Validation of Results}

The literature survey clearly and obviously showed that the static bifurcation buckling of a piezo-flexomagnetic nanobeam has not been studied thus far. Therefore, the only path to validate correctness of the formulation is neglecting piezomagneticity, flexomagneticity, and the strain gradient 
model to compare the results with a nano-sized circular beam $[67,68]$. Both parts of the literature employed a classical beam, however, ref. [67] used the differential transform solution method and ref. [68] used an explicit solution method. As can be shown from Table 3, our results are entirely matched with the literature.

Table 3. Critical loads' validation with literature $(E=1 \mathrm{TPa}, v=0.19, \mathrm{~d}=1 \mathrm{~nm})$.

\begin{tabular}{|c|c|c|c|c|c|c|c|c|c|}
\hline \multicolumn{10}{|c|}{$P_{C r}(n N)$} \\
\hline \multirow{2}{*}{ L (nm) } & \multicolumn{3}{|c|}{$\mu=0 \mathrm{~nm}^{2}$} & \multicolumn{3}{|c|}{$\mu=1 \mathrm{~nm}^{2}$} & \multicolumn{3}{|c|}{$\mu=4 \mathrm{~nm}^{2}$} \\
\hline & [67] & [68] & Present & [67] & [68] & Present & [67] & [68] & Present \\
\hline 10 & 4.8447 & 4.8447 & 4.8447 & 4.4095 & 4.4095 & 4.4095 & 3.4735 & 3.4735 & 3.4735 \\
\hline 12 & 3.3644 & 3.3644 & 3.3644 & 3.1486 & 3.1486 & 3.1486 & 2.6405 & 2.6405 & 2.6405 \\
\hline 14 & 2.4718 & 2.4718 & 2.4718 & 2.3533 & 2.3533 & 2.3533 & 2.0574 & 2.0574 & 2.0574 \\
\hline 16 & 1.8925 & 1.8925 & 1.8925 & 1.8222 & 1.8222 & 1.8222 & 1.6396 & 1.6396 & 1.6396 \\
\hline 18 & 1.4953 & 1.4953 & 1.4953 & 1.4511 & 1.4511 & 1.4511 & 1.3329 & 1.3329 & 1.3329 \\
\hline 20 & 1.2112 & 1.2112 & 1.2112 & 1.182 & 1.182 & 1.182 & 1.1024 & 1.1024 & 1.1024 \\
\hline
\end{tabular}

\subsection{Stability Analysis}

Investigating the flexomagneticity effect on the stability of a nanostructure is the main goal of this work. Table 4 presents applied material properties $[40,41]$. To take a rational amount for the nonlocal parameter, $0.5 \mathrm{~nm}<e_{0} a<0.8 \mathrm{~nm}[69]$, and $0<e_{0} a \leq 2 \mathrm{~nm}[70,71]$, are utilized.

Table 4. Material parameters of the piezo-flexomagnetic nanobeam.

\begin{tabular}{c}
\hline $\mathrm{CoFe}_{2} \mathrm{O}_{4}$ \\
\hline$C_{11}=286 \mathrm{GPa}$ \\
$q_{31}=580.3 \mathrm{~N} / \mathrm{A} . \mathrm{m}$ \\
$a_{33}=1.57 \times 10^{-4} \mathrm{~N} / \mathrm{A}^{2}$ \\
$(\mathrm{~A}=$ Ampere $)$ \\
\hline
\end{tabular}

Given Figure 2a, the nonlocal coefficient variations are plotted for four nanobeam states. That is, first, we have just the usual nanobeam by eliminating magnetic effects; the latter is a state where we merely have the piezomagnetic effect and finally the third and fourth states are when we have both the piezo- and flexomagnetic effects but with two different values of flexomagnetic property. As is clear from the figure, increasing the numerical value of the nonlocal parameter reduces the critical loads in all four states. Thereby, one of the important results of this graph is that when we consider a positive magnetic field and we have the piezomagnetic as well as flexomagnetic effect, the nanobeam has greater stability against the axial membrane forces. Furthermore, while purely having the piezomagnetic effect, the least stability is observed for the magneto-mechanical nanobeams. Further investigation is needed and is shown by the next figures. On the other hand, Figure $2 b$ shows a comparison of both piezomagnetic and piezo-flexomagnetic nanoscale beams while the strain gradient parameter is the changeable factor of the horizontal axis of the figure. As it is clarified by Figure $2 b$, it is noteworthy to say that increasing the strain gradient parameter results in a decrease in difference between the results of the piezo-flexomagnetic nanobeam with the piezomagnetic nanobeam. It is worth underlining that this proximity pertains to the flexomagnetic feature. This means that the increase of the value of the length scale parameter leads to a stiffening effect; therefore, by increasing the values of this parameter, the critical load's results tend to each other in magnetic beams. It can be argued that the length scale parameter makes the flexomagnetic effect ineffective. Eventually, if the length scale parameter is a large numerical value, it can be stated that the flexomagnetic effect is nothing and all the magnetic nanobeams will respond similarly. 


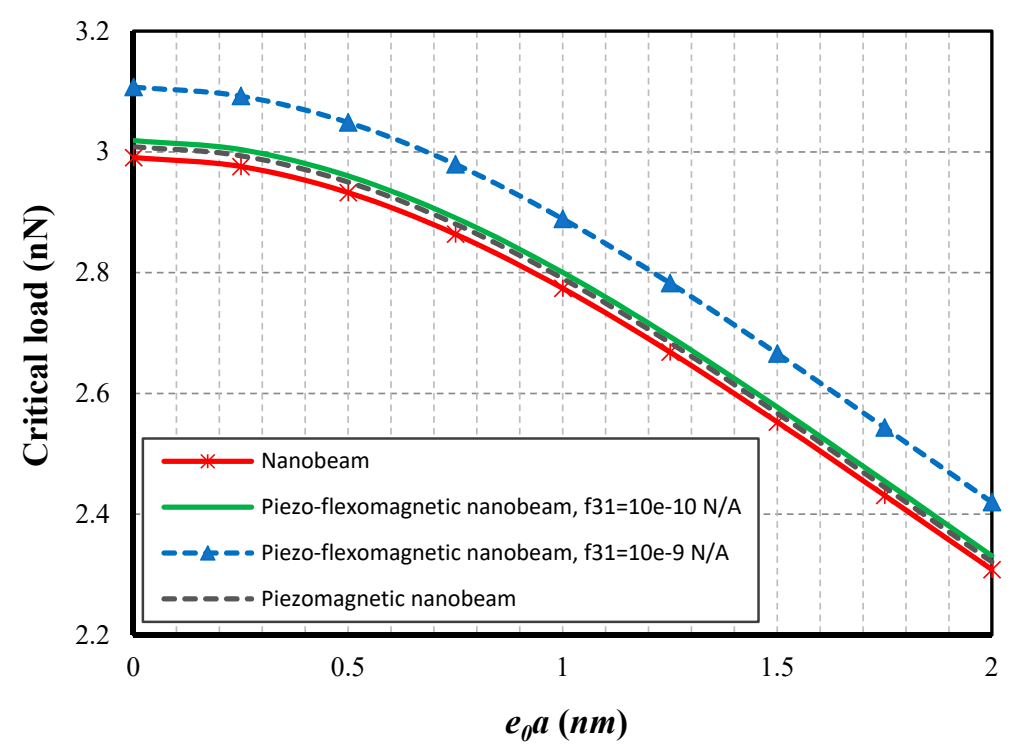

(a)

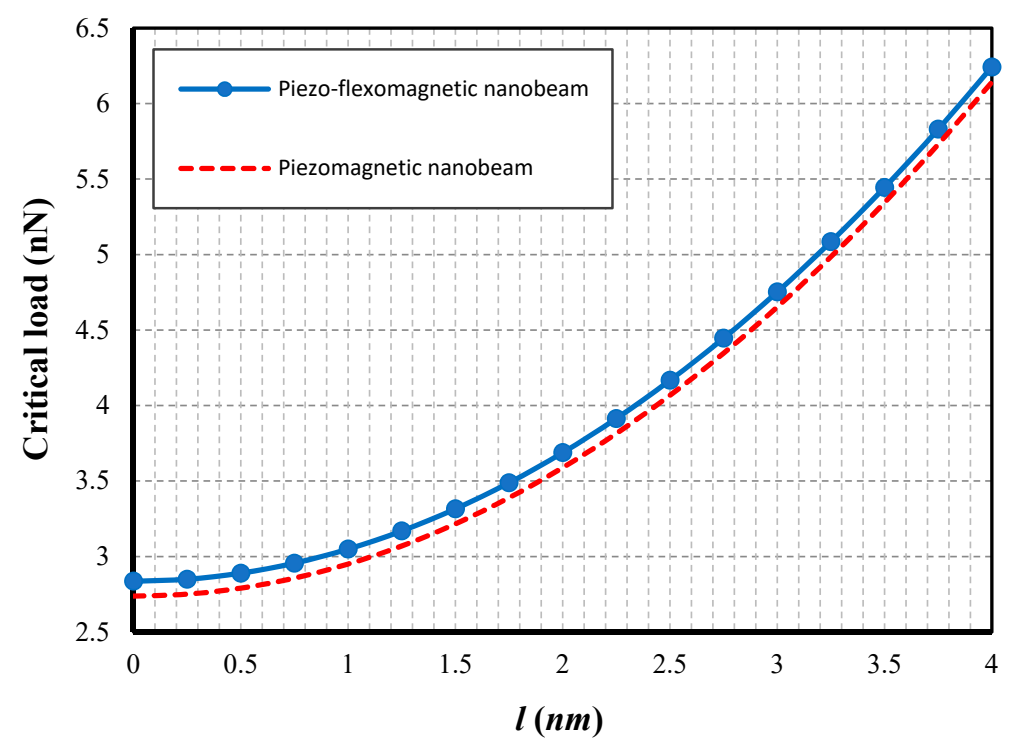

(b)

Figure 2. (a) Nonlocal parameter vs. four cases of non-porous nanobeams $(l=0.5 \mathrm{~nm}, L=10 \mathrm{~h}, \psi=1$ $\mathrm{mA})$. (b) The length scale strain gradient parameter vs. different cases of non-porous nanobeams $\left(e_{0} a=\right.$ $0.5 \mathrm{~nm}, L=10 \mathrm{~h}, \psi=1 \mathrm{~mA})$.

In Figure 3, we investigate the effects of given patterns of the porosities for two cases of the nano-sized magnetic beam, the first one with the flexomagnetic property (PFM) and the later one ignoring this physical feature. We observed in the previous figure that in attending the positive magnetic field, if the piezomagnetic nanobeam has a flexomagnetic property (piezo-flexomagnetic), it is the most stable case in terms of magneto-mechanical nanobeams. It can now be seen from this figure that for all PFM cases, the further in-plane resistance can be observed. Hence, one can prove that this effect makes material stiffer. Furthermore, increase of the value of the porosity parameter $(\alpha)$ leads to reduction of material stability in all cases. However, this decline is more noticeable for $\overline{\mathrm{X}}$ and $\overline{\mathrm{O}}$ porosities and insignificant for $\mathrm{O}$ and $\mathrm{X}$ samples. It is also notable that in terms of $\bar{X}$ and $\overline{\mathrm{O}}$ porosity 
types, an increase of value of the porosity parameter leads to more gaps between PFM and PM. This means that these kinds of porosities make the flexomagnetic property more important.

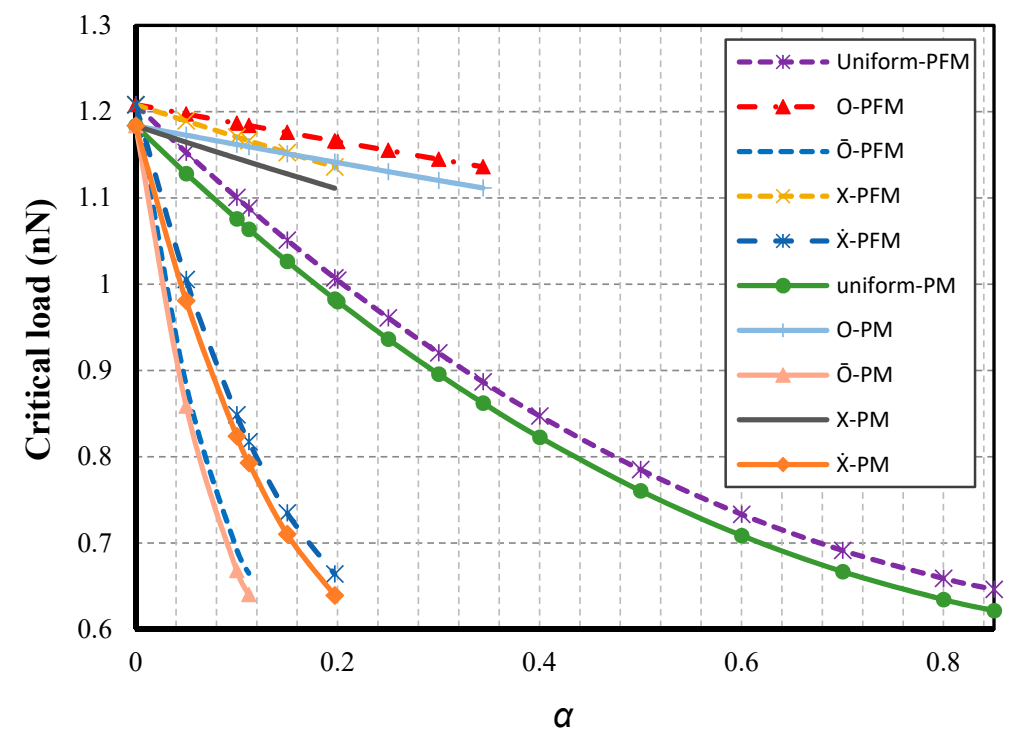

Figure 3. Types of porosity vs. two cases of nanobeams ( $\left.e_{0} a=0.5 \mathrm{~nm}, l=1 \mathrm{~nm}, L=20 \mathrm{~h}, \psi=1 \mathrm{~mA}\right)$.

Figure 4 is presented correspondingly to reveal the incremental variations of the external magnetic potential. In this figure, as in the previous ones, the nanobeam is investigated in different cases. As can be seen, the increased magnetization potential leads to greater stability of the magnetic nanobeams. Of importance in the diagram is the magnetic nanobeams containing lesser critical loads than the conventional nanobeam. As a matter of fact, the in-plane static stability for such beams in negative amounts of magnetic potential is smaller than that of the conventional nanobeam. This downward/upward trend is linear in the form of a steep slope, indicating that in very strong positive magnetic fields, the piezomagnetic effects and, especially the flexomagnetic ones, will be more and more pronounced. However, these effects are also significant and undeniable in a weak magnetic environment.

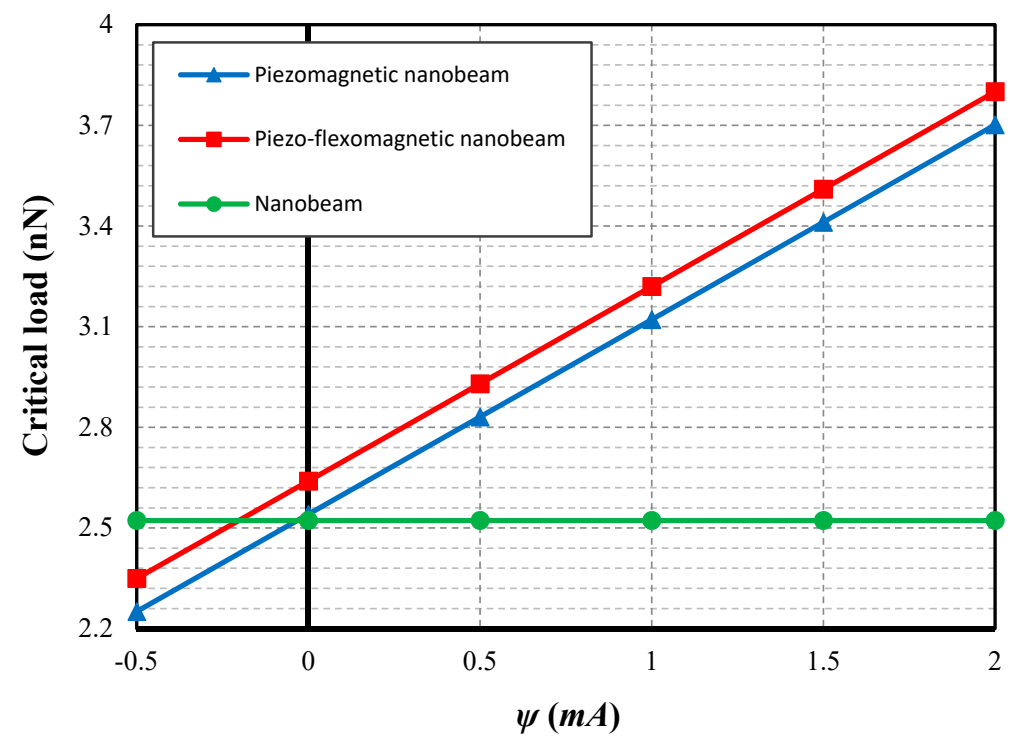

Figure 4. Magnetic potential vs. two cases of non-porous nanobeams $\left(e_{0} a=0.5 \mathrm{~nm}, l=1 \mathrm{~nm}, L=10 \mathrm{~h}\right)$. 
Figure 5 displays the aforementioned states of nanobeams concerning changes in their thicknesses. It can be inferred from the figure that at very small thicknesses, and also positive magnetic potential, the flexomagnetic property plays a vital role in in-plane stability. With the thickening of the nanobeams, the difference between the results of the piezomagnetic and piezo-flexomagnetic nanobeams decreases. Thereupon, for larger thicknesses, the flexomagnetic effect seems to be unimportant.

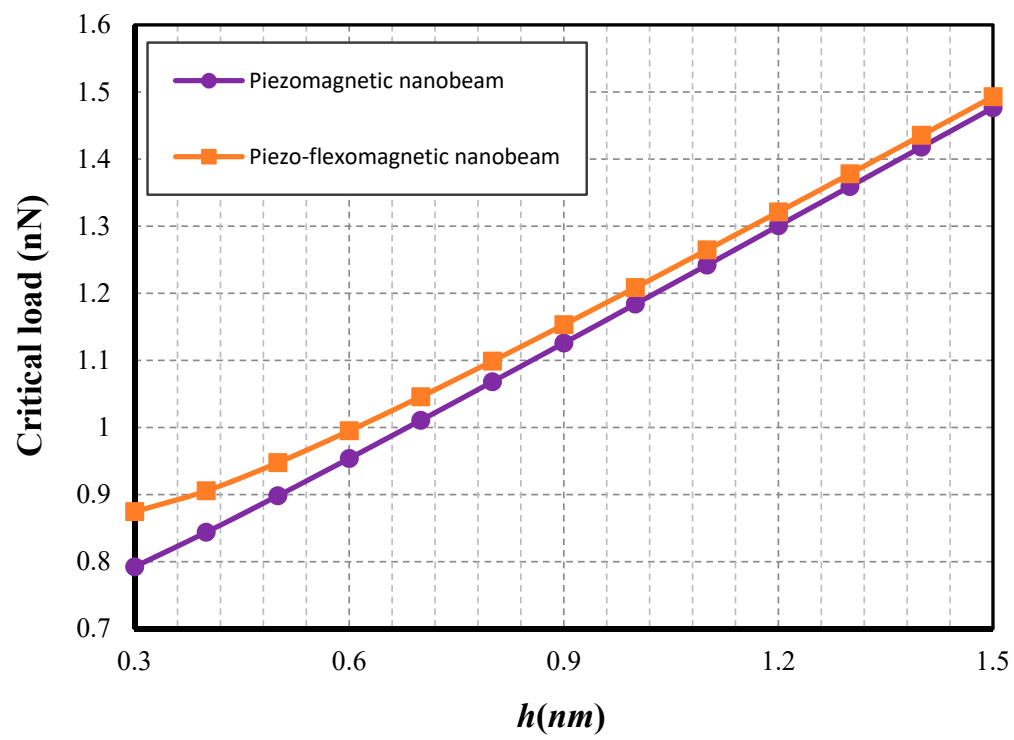

Figure 5. Thickness vs. two cases of non-porous nanobeams ( $\left.e_{0} a=0.5 \mathrm{~nm}, l=1 \mathrm{~nm}, \psi=1 \mathrm{~mA}, L=20 h\right)$.

\section{Conclusions}

This study presented the stability capacity of a porous nanobeam involving piezomagnetic as well as flexomagnetic impacts. To date, it is known that the mechanism of action of nanostructures is based on two principles of hardening and softening. This research applied these actions to a piezo-flexomagnetic nanobeam. Substituting Lagrangian, and nonlocal theory of strain gradient elasticity, the stability relation of the piezo-flexomagnetic nanobeam was gained. Thereafter, the Navier method gave a closed-form solution to reach numerical amounts of the in-plane static stability.

The flexomagnetic effect as a complex physical phenomenon into the magneto-mechanical coupling is known as a size-dependent property that was also here affected by small-scale parameters. Furthermore, the variations of thickness of the nanobeam affected the flexomagneticity, and this property is further noticeable for lower thicknesses of nanobeams. Moreover, comparing a piezomagnetic nanobeam with a piezo-flexomagnetic one showed that this feature presents more stable material. In addition, based on our observations, it was proved that despite the flexomagnetic effect being important in the lowest positive external magnetic potentials, if the potential is sufficiently large, the capacity of static stability for piezo-flexomagnetic nanobeams will be enhanced markedly. Another significant point obtained in this research work was the influence of porosity on the flexomagnetic response of the piezomagnetic nano-sized beam. The results showed that in some patterns of porosity, this imperfection can affect the flexomagnetic behavior of the material.

Author Contributions: Conceptualization, M.M., V.A.E. and K.K.Ż.; methodology, M.M., V.A.E. and K.K.Ż.; software, M.M.; validation, M.M.; formal analysis, M.M.; investigation, M.M. and V.A.E.; resources, M.M., V.A.E. and K.K.Ż.; data curation, M.M., V.A.E. and K.K.Ż.; writing—original draft preparation, M.M.; writing-review and editing, V.A.E. and K.K.Ż.; visualization, M.M.; supervision, V.A.E.; project administration, V.A.E.; funding acquisition, V.A.E. and K.K.Ż. All authors have read and agreed to the published version of the manuscript.

Funding: This research received no external funding. 
Acknowledgments: V.A.E. acknowledges the support of the Government of the Russian Federation (contract No. 14.Z50.31.0046). K.K.Ż. acknowledges the support of the Ministry of Science and Higher Education of Poland (project No. W/WM-IIM/3/2020).

Conflicts of Interest: The authors declare no conflict of interest. The authors declare that they have no known competing financial interests or personal relationships that could have appeared to influence the work reported in this paper.

\section{References}

1. Kabychenkov, A.F.; Lisovskii, F.V. Flexomagnetic and Flexoantiferromagnetic Effects in Centrosymmetric Antiferromagnetic Materials. Tech. Phys. 2019, 64, 980-983. [CrossRef]

2. Eliseev, E.A.; Morozovska, A.N.; Glinchuk, M.D.; Blinc, R. Spontaneous flexoelectric/flexomagnetic effect in nanoferroics. Phys. Rev. B 2009, 79, 165433. [CrossRef]

3. Lukashev, P.; Sabirianov, R.F. Flexomagnetic effect in frustrated triangular magnetic structures. Phys. Rev. B 2010, 82, 094417. [CrossRef]

4. Ma, W. Flexoelectricity: Strain gradient effects in ferroelectrics. Phys. Scr. 2007, T129, 180-183. [CrossRef]

5. Lee, D.; Yoon, A.; Jang, S.Y.; Yoon, J.-G.; Chung, J.-S.; Kim, M.; Scott, J.F.; Noh, T.W. Giant Flexoelectric Effect in Ferroelectric Epitaxial Thin Films. Phys. Rev. Lett. 2011, 107, 057602. [CrossRef]

6. Nguyen, T.D.; Mao, S.; Yeh, Y.-W.; Purohit, P.K.; McAlpine, M.C. Nanoscale Flexoelectricity. Adv. Mater. 2013, 25, 946-974. [CrossRef]

7. Zubko, P.; Catalan, G.; Tagantsev, A.K. Flexoelectric Effect in Solids. Annu. Rev. Mater. Res. 2013, 43, $387-421$. [CrossRef]

8. Yudin, P.V.; Tagantsev, A.K. Fundamentals of flexoelectricity in solids. Nanotechnology 2013, $24,432001$. [CrossRef]

9. Jiang, X.; Huang, W.; Zhang, S.; Tagantsev, A.K. Flexoelectric nano-generators: Materials, structures and devices. Nano Energy 2013, 2, 1079-1092. [CrossRef]

10. Yurkov, A.S.; Tagantsev, A.K. Strong surface effect on direct bulk flexoelectric response in solids. Appl. Phys. Lett. 2016, 108, 022904. [CrossRef]

11. Wang, B.; Gu, Y.; Zhang, S.; Chen, L.-Q. Flexoelectricity in solids: Progress, challenges, and perspectives. Prog. Mater. Sci. 2019, 106, 100570. [CrossRef]

12. Cross, L.E. Flexoelectric effects: Charge separation in insulating solids subjected to elastic strain gradients. J. Mater. Sci. 2006, 41, 53-63. [CrossRef]

13. Ma, W.; Cross, L.E. Observation of the flexoelectric effect in relaxor $\mathrm{Pb}(\mathrm{Mg} 1 / 3 \mathrm{Nb} 2 / 3) \mathrm{O} 3$ ceramics. Appl. Phys. Lett. 2001, 78, 2920-2921. [CrossRef]

14. Ma, W.; Cross, L.E. Flexoelectricity of barium titanate. Appl. Phys. Lett. 2006, 88, 232902. [CrossRef]

15. Zubko, P.; Catalan, G.; Buckley, A.; Welche, P.R.L.; Scott, J.F. Strain-gradient-induced polarization in SrTiO3 single crystals. Phys. Rev. Lett. 2007, 99, 167601. [CrossRef] [PubMed]

16. Eremeyev, V.A.; Ganghoffer, J.-F.; Konopińska-Zmysłowska, V.; Uglov, N.S. Flexoelectricity and apparent piezoelectricity of a pantographic micro-bar. Int. J. Eng. Sci. 2020, 149, 103213. [CrossRef]

17. Samani, M.S.E.; Beni, Y.T. Size dependent thermo-mechanical buckling of the flexoelectric nanobeam. Mater. Res. Express 2018, 5, 085018. [CrossRef]

18. Malikan, M.; Eremeyev, V.A. On the Dynamics of a Visco-Piezo-Flexoelectric Nanobeam. Symmetry 2020, 12, 643. [CrossRef]

19. Singhal, A.; Sedighi, H.M.; Ebrahimi, F.; Kuznetsova, I. Comparative study of the flexoelectricity effect with a highly/weakly interface in distinct piezoelectric materials (PZT-2, PZT-4, PZT-5H, LiNbO3, BaTiO3). Waves Random Complex Media 2019, 1-19. [CrossRef]

20. Chen, W.; Yan, Z.; Wang, L. On mechanics of functionally graded hard-magnetic soft beams. Int. J. Eng. Sci. 2020, 157, 103391. [CrossRef]

21. Ke, L.-L.; Wang, Y.-S. Free vibration of size-dependent magneto-electro-elastic nanobeams based on the nonlocal theory. Phys. E Low-Dimens. Syst. Nanostruct. 2014, 63, 52-61. [CrossRef]

22. Ping, T. Nonlinear free vibration analysis of nanobeams under magnetic field based on nonlocal elasticity theory. J. Vibroeng. 2016, 18, 1912-1919. [CrossRef]

23. Baghani, M.; Mohammadi, M.; Farajpour, A. Dynamic and Stability Analysis of the Rotating Nanobeam in a Nonuniform Magnetic Field Considering the Surface Energy. Int. J. Appl. Mech. 2016, 8, 1650048. [CrossRef] 
24. Ebrahimi, F.; Barati, M.R. Porosity-dependent vibration analysis of piezo-magnetically actuated heterogeneous nanobeams. Mech. Syst. Signal Process. 2017, 93, 445-459. [CrossRef]

25. Zenkour, A.M.; Arefi, M.; AlShehri, N.A. Size-dependent analysis of a sandwich curved nanobeam integrated with piezomagnetic face-sheets. Results Phys. 2017, 7, 2172-2182. [CrossRef]

26. Sun, X.-P.; Hong, Y.-Z.; Dai, H.; Wang, L. Nonlinear frequency analysis of buckled nanobeams in the presence of longitudinal magnetic field. Acta Mech. Solida Sin. 2017, 30, 465-473. [CrossRef]

27. Arefi, M.; Zenkour, A.M. Transient sinusoidal shear deformation formulation of a size-dependent three-layer piezo-magnetic curved nanobeam. Acta Mech. 2017, 228, 3657-3674. [CrossRef]

28. Liu, H.; Yang, J. Vibration of FG magneto-electro-viscoelastic porous nanobeams on visco-Pasternak foundation. Compos. Part B Eng. 2018, 155, 244-256. [CrossRef]

29. Karami, B.; Shahsavari, D.; Li, L. Hygrothermal wave propagation in viscoelastic graphene under in-plane magnetic field based on nonlocal strain gradient theory. Phys. E Low-Dimens. Syst. Nanostruct. 2018, 97, 317-327. [CrossRef]

30. Arefi, M.; Arani, A.H.S. Higher order shear deformation bending results of a magnetoelectrothermoelastic functionally graded nanobeam in thermal, mechanical, electrical, and magnetic environments. Mech. Based Des. Struct. Mach. 2018, 46, 669-692. [CrossRef]

31. Alibeigi, B.; Beni, Y.T. On the size-dependent magneto/electromechanical buckling of nanobeams. Eur. Phys. J. Plus 2018, 133, 398. [CrossRef]

32. Azrar, A.; Ben Said, M.; Azrar, L.; Aljinaidi, A. Dynamic instability analysis of magneto-electro-elastic beams with uncertain parameters under static and parametric electric and magnetic fields. Compos. Struct. 2019, 226, 111185. [CrossRef]

33. Zhen, Y.-X.; Wen, S.-L.; Tang, Y. Free vibration analysis of viscoelastic nanotubes under longitudinal magnetic field based on nonlocal strain gradient Timoshenko beam model. Phys. E Low-Dimens. Syst. Nanostructures 2019, 105, 116-124. [CrossRef]

34. Arefi, M.; Kiani, M.; Civalek, Ö. 3-D magneto-electro-thermal analysis of layered nanoplate including porous core nanoplate and piezomagnetic face-sheets. Appl. Phys. A 2020, 126, 76. [CrossRef]

35. Mirjavadi, S.S.; Forsat, M.; Nikookar, M.; Barati, M.R.; Hamouda, A. Nonlinear forced vibrations of sandwich smart nanobeams with two-phase piezo-magnetic face sheets. Eur. Phys. J. Plus 2019, 134, 508. [CrossRef]

36. Mirjavadi, S.S.; Forsat, M.; Barati, M.R.; Abdella, G.M.; Hamouda, A.M.S.; Afshari, B.M.; Rabby, S. Post-buckling analysis of piezo-magnetic nanobeams with geometrical imperfection and different piezoelectric contents. Microsyst. Technol. 2019, 25, 3477-3488. [CrossRef]

37. Jalaei, M.; CivalekÖ. On dynamic instability of magnetically embedded viscoelastic porous FG nanobeam. Int. J. Eng. Sci. 2019, 143, 14-32. [CrossRef]

38. Ghane, M.; Saidi, A.R.; Bahaadini, R. Vibration of fluid-conveying nanotubes subjected to magnetic field based on the thin-walled Timoshenko beam theory. Appl. Math. Model. 2020, 80, 65-83. [CrossRef]

39. Żur, K.; Arefi, M.; Kim, J.; Reddy, J. Free vibration and buckling analyses of magneto-electro-elastic FGM nanoplates based on nonlocal modified higher-order sinusoidal shear deformation theory. Compos. Part B Eng. 2020, 182, 107601. [CrossRef]

40. Sidhardh, S.; Ray, M. Flexomagnetic response of nanostructures. J. Appl. Phys. 2018, 124, 244101. [CrossRef]

41. Zhang, N.; Zheng, S.; Chen, D. Size-dependent static bending of flexomagnetic nanobeams. J. Appl. Phys. 2019, 126, 223901. [CrossRef]

42. Malikan, M.; Eremeyev, V.A. Free Vibration of Flexomagnetic Nanostructured Tubes Based on Stress-driven Nonlocal Elasticity. In Engineering Design Applications; Springer Science and Business Media LLC.: Berlin/Heidelberg, Germany, 2020; Volume 134, pp. 215-226.

43. Malikan, M.; Eremeyev, V.A. On the geometrically nonlinear vibration of a piezo-flexomagnetic nanotube. Math. Methods Appl. Sci. 2020. [CrossRef]

44. Malikan, M.; Eremeyev, V.A. On Nonlinear Bending Study of a Piezo-Flexomagnetic Nanobeam Based on an Analytical-Numerical Solution. Nanomaterials 2020, 10, 1762. [CrossRef] [PubMed]

45. Malikan, M.; Uglov, N.S.; Eremeyev, V.A. On instabilities and post-buckling of piezomagnetic and flexomagnetic nanostructures. Int. J. Eng. Sci. 2020, 157, 103395. [CrossRef]

46. Song, X.; Li, S.-R. Thermal buckling and post-buckling of pinned-fixed Euler-Bernoulli beams on an elastic foundation. Mech. Res. Commun. 2007, 34, 164-171. [CrossRef] 
47. Reddy, J. Nonlocal nonlinear formulations for bending of classical and shear deformation theories of beams and plates. Int. J. Eng. Sci. 2010, 48, 1507-1518. [CrossRef]

48. Barretta, R.; Feo, L.; Luciano, R.; De Sciarra, F.M. Variational formulations for functionally graded nonlocal Bernoulli-Euler nanobeams. Compos. Struct. 2015, 129, 80-89. [CrossRef]

49. Akgöz, B.; Civalek, Ö. Buckling analysis of functionally graded microbeams based on the strain gradient theory. Acta Mech. 2013, 224, 2185-2201. [CrossRef]

50. Sarparast, H.; Ebrahimi-Mamaghani, A.; Safarpour, M.; Ouakad, H.M.; Dimitri, R.; Tornabene, F. Nonlocal study of the vibration and stability response of small-scale axially moving supported beams on viscoelastic-Pasternak foundation in a hygro-thermal environment. Math. Methods Appl. Sci. 2020. [CrossRef]

51. Lim, C.; Zhang, G.; Reddy, J. A higher-order nonlocal elasticity and strain gradient theory and its applications in wave propagation. J. Mech. Phys. Solids 2015, 78, 298-313. [CrossRef]

52. Ansari, R.; Sahmani, S.; Arash, B. Nonlocal plate model for free vibrations of single-layered graphene sheets. Phys. Lett. A 2010, 375, 53-62. [CrossRef]

53. Khorshidi, M.A. The material length scale parameter used in couple stress theories is not a material constant. Int. J. Eng. Sci. 2018, 133, 15-25. [CrossRef]

54. She, G.L. Wave propagation of FG polymer composite nanoplates reinforced with GNPs. Steel Compos. Struct. 2020, 37, 27-35.

55. She, G.L.; Liu, H.B.; Karami, B. On resonance behavior of porous FG curved nanobeams. Steel Compos. Struct. 2020, 36, 179-186.

56. Malikan, M.; Dimitri, R.; Tornabene, F. Transient response of oscillated carbon nanotubes with an internal and external damping. Compos. Part B Eng. 2019, 158, 198-205. [CrossRef]

57. Malikan, M.; Krasheninnikov, M.; Eremeyev, V.A. Torsional stability capacity of a nano-composite shell based on a nonlocal strain gradient shell model under a three-dimensional magnetic field. Int. J. Eng. Sci. 2020, 148, 103210. [CrossRef]

58. Malikan, M. On the plastic buckling of curved carbon nanotubes. Theor. Appl. Mech. Lett. 2020, 10, 46-56. [CrossRef]

59. Malikan, M.; Eremeyev, V.A. Post-critical buckling of truncated conical carbon nanotubes considering surface effects embedding in a nonlinear Winkler substrate using the Rayleigh-Ritz method. Mater. Res. Express 2020, 7, 025005. [CrossRef]

60. Karami, B.; Janghorban, M.; Rabczuk, T. Dynamics of two-dimensional functionally graded tapered Timoshenko nanobeam in thermal environment using nonlocal strain gradient theory. Compos. Part B Eng. 2020, 182, 107622. [CrossRef]

61. Apuzzo, A.; Barretta, R.; Faghidian, S.A.; Luciano, R.; De Sciarra, F.M. Nonlocal strain gradient exact solutions for functionally graded inflected nano-beams. Compos. Part B Eng. 2019, 164, 667-674. [CrossRef]

62. Tang, H.; Li, L.; Hu, Y. Buckling analysis of two-directionally porous beam. Aerosp. Sci. Technol. 2018, 78, 471-479. [CrossRef]

63. Barretta, R.; De Sciarra, F.M. Constitutive boundary conditions for nonlocal strain gradient elastic nano-beams. Int. J. Eng. Sci. 2018, 130, 187-198. [CrossRef]

64. Barretta, R.; De Sciarra, F.M. Variational nonlocal gradient elasticity for nano-beams. Int. J. Eng. Sci. 2019, 143, 73-91. [CrossRef]

65. De Sciarra, F.M.; Barretta, R. A new nonlocal bending model for Euler-Bernoulli nanobeams. Mech. Res. Commun. 2014, 62, 25-30. [CrossRef]

66. Romano, G.; Barretta, R.; Diaco, M.; De Sciarra, F.M. Constitutive boundary conditions and paradoxes in nonlocal elastic nanobeams. Int. J. Mech. Sci. 2017, 121, 151-156. [CrossRef]

67. Pradhan, S.; Reddy, G. Buckling analysis of single walled carbon nanotube on Winkler foundation using nonlocal elasticity theory and DTM. Comput. Mater. Sci. 2011, 50, 1052-1056. [CrossRef]

68. Wang, C.M.; Zhang, Y.Y.; Ramesh, S.S.; Kitipornchai, S. Buckling analysis of micro- and nano-rods/tubes based on nonlocal Timoshenko beam theory. J. Phys. D Appl. Phys. 2006, 39, 3904-3909. [CrossRef]

69. Ansari, R.; Sahmani, S.; Rouhi, H. Rayleigh-Ritz axial buckling analysis of single-walled carbon nanotubes with different boundary conditions. Phys. Lett. A 2011, 375, 1255-1263. [CrossRef] 
70. Duan, W.H.; Wang, C.M. Exact solutions for axisymmetric bending of micro/nanoscale circular plates based on nonlocal plate theory. Nanotechnology 2007, 18, 385704. [CrossRef]

71. Duan, W.H.; Wang, C.M.; Zhang, Y.Y. Calibration of nonlocal scaling effect parameter for free vibration of carbon nanotubes by molecular dynamics. J. Appl. Phys. 2007, 101, 024305. [CrossRef]

Publisher's Note: MDPI stays neutral with regard to jurisdictional claims in published maps and institutional affiliations.

(C) 2020 by the authors. Licensee MDPI, Basel, Switzerland. This article is an open access article distributed under the terms and conditions of the Creative Commons Attribution (CC BY) license (http://creativecommons.org/licenses/by/4.0/). 